\title{
Epidemiological trend in inflammatory bowel disease in Taiwan from 2001 to 2015: a nationwide population- based study
}

\author{
Hsu-Heng Yen ${ }^{1,2}$, Meng-Tzu Weng ${ }^{3}$, Chien-Chih Tung ${ }^{4}$, Yu-Ting Wang ${ }^{5}$, Yuan Ting Chang ${ }^{6}$, Chin-Hao Chang ${ }^{5,6}$, \\ Ming-Jium Shieh ${ }^{7}$, Jau-Min Wong ${ }^{8}$, Shu-Chen Wei ${ }^{1,9}$ \\ ${ }^{1}$ Department of Internal Medicine and ${ }^{2}$ Endoscopy Center, Changhua Christian Hospital, Changhua; ${ }^{3}$ Department of Internal Medicine, Far \\ Eastern Memorial Hospital, New Taipei; ${ }^{4}$ Department of Integrated Diagnostics \& Therapeutics and ${ }^{5}$ Medical Research, National Taiwan \\ University Hospital, Taipei; ${ }^{6}$ Department of Public Health, National Taiwan University, Taipei; Departments of ${ }^{7}$ Oncology and ${ }^{8}$ Internal \\ Medicine and ${ }^{9}$ Inflammatory Bowel Disease Clinical and Study Integrated Center, National Taiwan University Hospital, Taipei, Taiwan
}

Background/Aims: Incidences of inflammatory bowel disease (IBD), ulcerative colitis (UC), and Crohn's disease (CD), have been increasing in Asia. In this study, we report the relevant clinical characteristics and determined the epidemiological trend of IBD in Taiwan from 2001 to 2015. Methods: A retrospective study was conducted to analyze data recorded from January 2001 through December 2015 in the registered database compiled by the National Health Insurance and provided by the Ministry of Health and Welfare, Taiwan. Results: A total of 3,806 patients with catastrophic IBD illness were registered from 2001 to 2015 in Taiwan (CD, 919; UC, 2,887). The crude incidence of CD increased from $0.17 / 100,000$ in 2001 to $0.47 / 100,000$ in 2015, whereas that of UC increased from 0.54/100,000 in 2001 to $0.95 / 100,000$ in 2015. The prevalence of CD increased from 0.6/100,000 in 2001 to 3.9/100,000 in 2015, whereas that of UC increased from 2.1/100,000 in 2001 to 12.8/100,000 in 2015. The male-to-female ratio in the study sample was 2.19 for CD and 1.62 for UC. The median age of those registered with CD was lower than that of those registered for UC: 38.86 and 44.86 years, respectively. A significantly greater increase in CD incidence rate was identified among 20 to 39-year-old compared with other age groups. Conclusions: Using Taiwan's nationwide insurance database, we determined that the number of patients with CD increased more rapidly during the study period than the number of patients with UC, especially among age 20 to 39-year-old, resulting in a decreased UC-to-CD ratio. (Intest Res 2019;17:54-62)

Key Words: Crohn disease; Colitis, ulcerative; Incidence; Prevalence; Taiwan

\section{INTRODUCTION}

Inflammatory bowel disease (IBD), including CD and UC, are common chronic GI diseases in Western countries. ${ }^{1-4}$ IBD has been increasing in both incidence and prevalence in many newly industrialized countries since the beginning of the 21 st

Received June 27, 2018. Revised October 6, 2018. Accepted October 6, 2018 Correspondence to Shu-Chen Wei, Inflammatory Bowel Disease Clinical and Study Integrated Center, National Taiwan University Hospital, No. 7 Chung-Shan South Road, Taipei, Taiwan. Tel: +886-23123456 (ext. 65768), Fax:+886-23947927,E-mail: shuchenwei@ntu.edu.tw

ORCID Hsu-Heng Yen (https://orcid.org/0000-0002-3494-2245), Shu-Chen Wei (https://orcid.org/0000-0002-5017-5840) century. ${ }^{1,3,5-8}$ The reason for the global increase in this initially Western-prevalent disease has not been established but is most likely related to environment factors, including improved hygiene, civilization, and the spread of Western lifestyles and diets. $^{1}$

Longitudinal studies of Western populations performed within the past 50 years have revealed a pattern of initial increases in UC incidence, followed by increases in CD incidence at various intervals over the specified decades. ${ }^{3,9}$ For example, a study in Denmark ${ }^{10}$ reported a steady increase in CD incidence but stable UC incidence from 1981 to 1992. In Japan, which was one of the first among Eastern countries to 
adopt aspects of Western lifestyles, researchers reported a rapid rise of the incidence rate of UC beginning in 1970, with a peak in in 1974, followed by a peak in the CD incidence rate in 1979. ${ }^{11}$ This trend was also observed in other low-incidence Asian countries such as Hong Kong (from 8.94 in 1981-1985 to 1.03 in 2011-2014), ${ }^{12}$ South Korea (from 6.8 in 1986-1990 to 2.3 in 2001-2005), ${ }^{13}$ and Malaysia (from 8:1 in 1990-2000 to $3.6: 1$ in 2000-2010). ${ }^{14}$

Western studies have reported that CD and UC are more prevalent among female patients or that no intersex difference exists. ${ }^{15}$ By contrast, the data from our study as well as reports from other Asian countries have indicated that IBDs, especially CD, are more prevalent among male patients. ${ }^{5}$ Although the exact reason for these disparate patterns is unknown, a difference in genetic background between Asian and Western populations ${ }^{16}$ may be a contributing factor, and the male-preference culture in Asian countries may lead to differences for male and female babies regarding breastfeeding, antibiotic use in childhood, and subsequent development of IBDs. ${ }^{17}$

In Taiwan, the first case of IBD was diagnosed in 1969 as UC at the National Taiwan University Hospital; ${ }^{18}$ however, no reliable nationwide descriptive epidemiology data ${ }^{19}$ from the 20 century regarding IBD in Taiwan is available. ${ }^{5}$ Since 1997, IBDs, including CD and UC, have been registered as catastrophic illnesses due to their potential to cause repeated admissions and their requirements for chronic, sensitive care. Accordingly, medical costs are covered by insurance for patients registered with relevant diagnoses. Submission of a clinical diagnosis, pathological report, and possibly endoscopic or radiologic image studies for formal review by experts is required to validate and register the patient's diagnosis of $\mathrm{CD}$ or UC. In our previous study, we demonstrated that CD and UC increased in both incidence and prevalence in Taiwan from 1998 to 2008, according to the nationwide insurance registration data. ${ }^{5,8}$ In this study, we used the same nationwide database and now provide an updated report on the incidence, prevalence, and epidemiological trend of IBD in Taiwanese patients since the beginning of the 21 st century.

\section{METHODS}

\section{Data Source and Ethical Considerations}

Data from January 2001 to December 2015 were compiled for this nationwide population-based study of IBD. The National Health Insurance (NHI) is a compulsory program with coverage rate of $99.7 \%$ of the population in Taiwan. The NHI claims data provide clinical information including prescription, diagnoses, and hospitalizations for population-based epidemiologic research (https://dep.mohw.gov.tw/DOS). Under strict confidentiality guidelines and in accordance with personal electronic data protection regulations, the study population were obtained from NHI data (including Catastrophic Illness Patients Registry and Taiwan Cancer Registry), department of statistics of the Ministry of Health and Welfare, Taiwan. The present study was approved by the Ethics Review Board of the National Taiwan University Hospital (IRB No. 201507018W) and informed consent was waived.

\section{Patient Identification}

The Ministry of Health and Welfare has classified UC and CD as catastrophic illnesses that require life-long treatment. Patients with a diagnosis of UC or CD generally submit relevant information (including their clinical information, imaging studies, endoscopic studies, and pathology reports) to the NHI program when applying for a catastrophic-illness certificate. After receiving the clinical information, the reviewers of the NHI approve the certification when the diagnosis meets the IBD diagnostic criteria. ${ }^{20,21}$ We used diagnostic codes (International Classification of Diseases, Ninth Revision, Clinical Modification 2001 edition) to retrieve the data of patients with IBDs (UC, 556.XX; CD, 555.XX) from the catastrophic-illnessregistration database. As described, the NHI claim database collects the medical records and information of each registered patient, including sex, date of birth, and date of catastrophic-illness registration.

\section{Statistical Analyses}

The characteristics of the studied group of patients with IBD, including year of registration, sex ratio, and age distribution, are described in this paper. Annual crude incidence and prevalence were defined as the number of newly diagnosed patients and number of patients with IBD per 100,000 persons per year, respectively. All statistical analyses were performed using SAS version 9.4 (SAS Institute, Cary, NC, USA). A $P$-value of less than 0.05 indicated statistical significance.

\section{RESULTS}

\section{Incidence and Prevalence of IBD Increased Steadily from 2001 to 2015}

A total of 3,806 patients with IBD were registered from 2001 to 2015 in Taiwan. Among them, 919 were diagnosed with CD 
and 2,887 with UC (Table 1). As illustrated in Fig. 1, the crude incidence of CD increased from 0.17/100,000 in 2001 to $0.47 / 100,000$ in 2015 . The crude incidence of UC increased from $0.54 / 100,000$ in 2001 to $0.95 / 100,000$ in 2015 . The joinpoint trend analysis for the general trends in patients diagnosed with IBD were significant from 2001 to 2015 , with the annual percent change (APC) as 2.69. The trend of CD also increased significantly according to the joinpoint trend analysis from

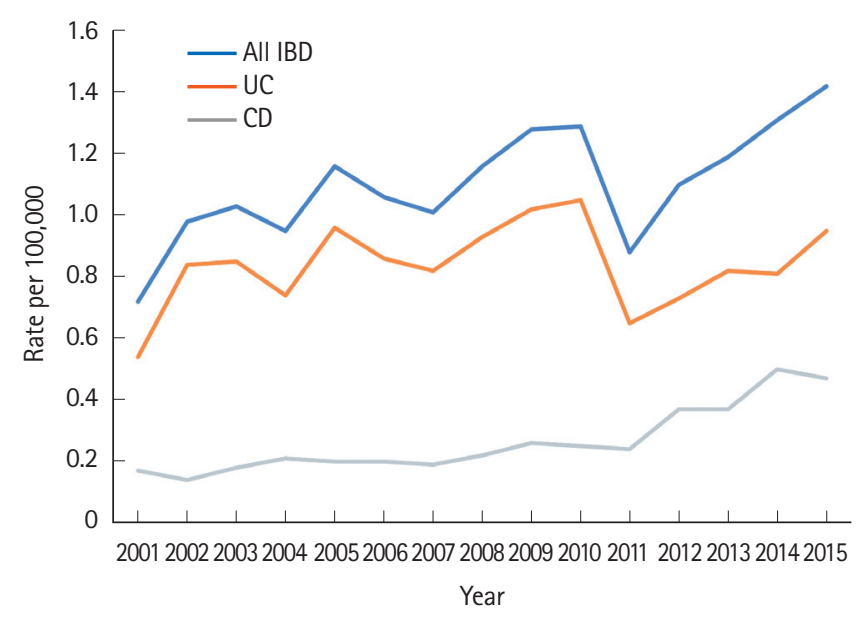

Fig. 1. Incidence of IBD from 2001 to 2015 in Taiwan.
2001 to 2015, with the APCs as 4.75 (2001-2010) and 15.92 (2010-2015); whereas they were insignificant of the trend for UC, since the APC value was 0.82 from 2001 to 2015. The crude prevalence of CD increased from 0.6/100,000 in 2001 to $3.9 / 100,000$ in 2015. The crude prevalence of UC increased from 2.1/100,000 in 2001 to 12.8/100,000 in 2015 (Fig. 2).

The study period was arbitrarily divided into the 3 stages: 2001 to 2005; 2006 to 2010; and 2011 to 2015 (Table 2) for

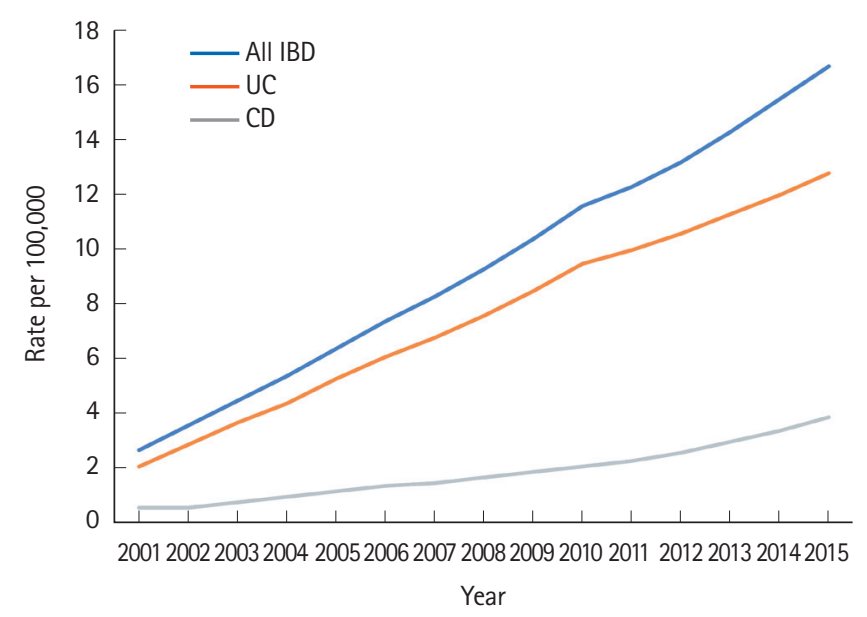

Fig. 2. Prevalence of IBD from 2001 to 2015 in Taiwan.

Table 1. The Incidence Rate of IBD, CD, and UC from 2001 to 2015

\begin{tabular}{|c|c|c|c|c|c|c|c|}
\hline \multirow{2}{*}{ Year } & \multirow{2}{*}{ Population } & \multicolumn{2}{|c|}{ IBD } & \multicolumn{2}{|c|}{$C D$} & \multicolumn{2}{|c|}{ UC } \\
\hline & & No. & IR & No. & IR & No. & IR \\
\hline 2001 & $22,341,120$ & 160 & 0.72 & 39 & 0.17 & 121 & 0.54 \\
\hline 2002 & $22,463,172$ & 220 & 0.98 & 32 & 0.14 & 188 & 0.84 \\
\hline 2003 & $22,562,663$ & 233 & 1.03 & 41 & 0.18 & 192 & 0.85 \\
\hline 2004 & $22,646,836$ & 215 & 0.95 & 48 & 0.21 & 167 & 0.74 \\
\hline 2006 & $22,823,455$ & 243 & 1.06 & 46 & 0.20 & 197 & 0.86 \\
\hline 2007 & $22,917,444$ & 231 & 1.01 & 43 & 0.19 & 188 & 0.82 \\
\hline 2008 & $22,997,696$ & 266 & 1.16 & 51 & 0.22 & 215 & 0.93 \\
\hline 2009 & $23,078,402$ & 296 & 1.28 & 61 & 0.26 & 235 & 1.02 \\
\hline 2013 & $23,344,670$ & 278 & 1.19 & 86 & 0.37 & 192 & 0.82 \\
\hline 2014 & $23,403,635$ & 307 & 1.31 & 118 & 0.50 & 189 & 0.81 \\
\hline 2015 & $23,462,914$ & 334 & 1.42 & 111 & 0.47 & 223 & 0.95 \\
\hline Total & & 3,806 & & 919 & & 2,887 & \\
\hline
\end{tabular}

$\mathrm{IR}$, incidence rate. 
newly registered IBD. The overall incidence and prevalence of IBD significantly increased over the 3 stages. Additionally, both the incidence of CD $(0.18 / 100,000,0.22 / 100,000$, and
0.39/100,000; $P<0.0001)$ and prevalence of CD $(0.84 / 100,000$, $1.71 / 100,000$, and $3.04 / 100,000 ; P<0.0001)$ increased significantly over the 3 stages (Table 3 ). Finally, although the differ-

Table 2. Clinical Features of Patients with Newly Registered IBD in 2001 to 2015

\begin{tabular}{|c|c|c|c|c|c|}
\hline All IBD & Over all & $\begin{array}{c}\text { Stage } 1 \\
(2001-2005)\end{array}$ & $\begin{array}{c}\text { Stage } 2 \\
(2006-2010)\end{array}$ & $\begin{array}{c}\text { Stage } 3 \\
(2011-2015)\end{array}$ & $P$-value \\
\hline No. of cases (UC/CD) & $2,887 / 919$ & $886 / 205$ & $835 / 201$ & $1,166 / 513$ & - \\
\hline \multicolumn{6}{|l|}{ Age (yr) } \\
\hline Mean & $43.29 \pm 16.58$ & $43.98 \pm 17.00$ & $43.46 \pm 16.22$ & $42.74 \pm 16.62$ & 0.1478 \\
\hline Median (01-03) & $42(31-54)$ & $43(32-55)$ & $42(32-54)$ & $43(30-55)$ & \\
\hline Age distribution & & & & & 0.5689 \\
\hline$<20$ & $243(6.38)$ & $67(6.14)$ & $58(5.60)$ & $118(7.03)$ & \\
\hline $20-39$ & $1,418(37.26)$ & $387(35.47)$ & 406 (39.19) & $625(37.22)$ & \\
\hline $40-59$ & $1,492(39.2)$ & 434 (39.78) & 406 (39.19) & $652(38.83)$ & \\
\hline $60-79$ & $592(15.55)$ & $185(16.96)$ & $149(14.38)$ & $258(15.37)$ & \\
\hline$\geq 80$ & $61(1.60)$ & 18 (1.65) & $17(1.64)$ & $26(1.55)$ & \\
\hline Male/female ratio & 1.74 & 1.73 & 1.59 & 1.86 & 0.1663 \\
\hline Prevalence $(1 / 100,000)$ & 9.50 & 4.54 & 9.39 & 14.40 & $<0.0001$ \\
\hline Incidence $(1 / 100,000)$ & 1.11 & 0.97 & 1.16 & 1.18 & $<0.0001$ \\
\hline
\end{tabular}

Values are presented as mean $\pm \mathrm{SD}$, median (range), or number (\%).

Table 3. Clinical Features of Patients with CD in 2001 to 2015

\begin{tabular}{|c|c|c|c|c|c|}
\hline $\mathrm{CD}$ & Over all & $\begin{array}{c}\text { Stage } 1 \\
(2001-2005)\end{array}$ & $\begin{array}{c}\text { Stage } 2 \\
(2006-2010)\end{array}$ & $\begin{array}{c}\text { Stage } 3 \\
(2011-2015)\end{array}$ & $P$-value \\
\hline No. of cases & 919 & 205 & 201 & 513 & - \\
\hline \multicolumn{6}{|l|}{ Age (yr) } \\
\hline Mean & $37.95 \pm 18.41$ & $37.83 \pm 19.94$ & $38.52 \pm 18.34$ & $38.03 \pm 17.93$ & 0.9249 \\
\hline Median (01-03) & $35(24-50)$ & $37(23-51)$ & $36(25-50)$ & $34(25-50)$ & \\
\hline \multicolumn{6}{|l|}{ Peak age (top 5 age, \%) } \\
\hline & 25 (2.99) & $0(4.39)$ & $25(4.48)$ & 30 (3.70) & \\
\hline & $32(2.89)$ & 39 (3.90) & 23 (3.98) & $29(3.12)$ & \\
\hline & $29(2.69)$ & $20(3.41)$ & $18(3.48)$ & 27 (2.92) & \\
\hline & $22(2.59)$ & $22(3.41)$ & $26(3.48)$ & $25(2.73)$ & \\
\hline & $30(2.49)$ & 37 (2.93) & 33 (2.99) & $31(2.73)$ & \\
\hline Age groups & & & & & 0.7740 \\
\hline$<20$ & 128 (13.93) & 34 (16.59) & $27(13.43)$ & $67(13.06)$ & \\
\hline $20-39$ & $415(45.16)$ & 81 (39.51) & $92(45.77)$ & $242(47.17)$ & \\
\hline $40-59$ & $241(26.22)$ & $61(29.76)$ & $53(26.37)$ & $127(24.76)$ & \\
\hline $60-79$ & $115(12.51)$ & $25(12.20)$ & $25(12.44)$ & 65 (12.67) & \\
\hline$\geq 80$ & $20(2.18)$ & $4(1.95)$ & $4(1.99)$ & $12(2.34)$ & \\
\hline Male/female ratio & 2.19 & 2.25 & 2.72 & 2.00 & 0.2402 \\
\hline Prevalence $(1 / 100,000)$ & 1.88 & 0.84 & 1.71 & 3.04 & $<0.0001$ \\
\hline Incidence $(1 / 100,000)$ & 0.27 & 0.18 & 0.22 & 0.39 & $<0.0001$ \\
\hline
\end{tabular}

Values are presented as mean $\pm S D$, median (range), or number (\%). 
ence was less significant, the incidence of UC $(0.79 / 100,000$, $0.94 / 100,000$, and $0.79 / 100,000 ; P<0.0001)$ and prevalence of UC $(3.70 / 100,000,7.68 / 100,000$, and $11.36 / 100,000 ; P<0.0001)$ also increased significantly over the 3 stages (Table 4 ).

To clarify the possible factors that contributed to this change, we grouped the patients with CD according to age, and we then discovered that the increase in CD was mostly concentrated in individuals aged 20 to 39 years (Fig. 3).

\section{UC-to-CD Incidence Ratio Decreased from 2001 to 2015 in Taiwan}

We then compared the incidence ratio of UC to CD (Fig. 4) from 2001 to 2015 and determined that it significantly decreased from 4.32 to 4.17 and finally to 2.03 across the 3 stages.

\section{Sex and Age Distribution Trends}

A male-predominant pattern for IBD in Taiwan, and more so for CD than for UC, was evident across all 3 stages. The overall male-to-female ratio was 2.19 for CD and 1.62 for UC. The male-to-female ratios of CD and UC were steady over the 3 stages from 2001 to 2015 (Fig. 5).
The mean ages of registration for IBD, CD, and UC from 2001 to 2015 were 43.29, 37.95, and 44.94 years, respectively. No significant change was evident regarding the median ages

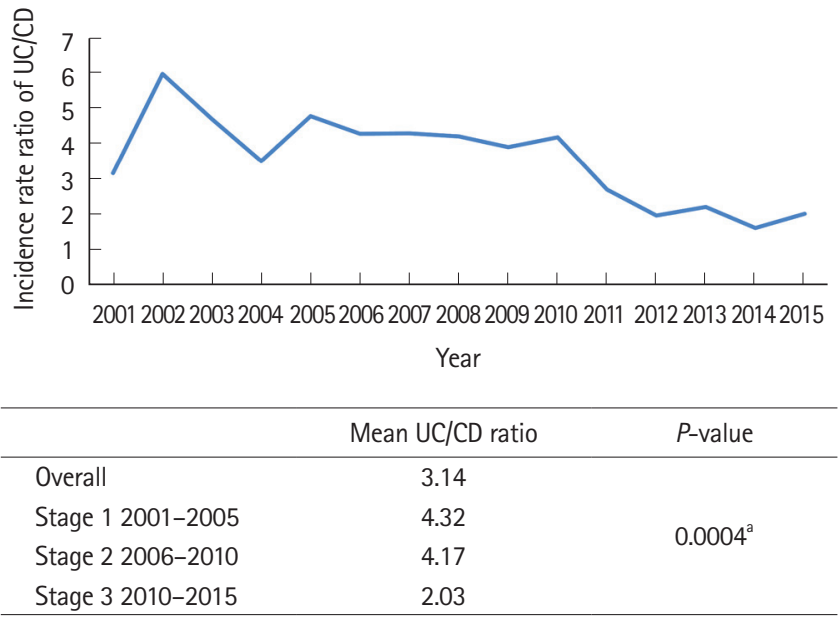

Fig. 3. Incidence rate ratio of $U C$ to $C D$ over time. Comparisons between stages 3 and 1 and stages 3 and 2 revealed significant differences. No significant difference was observed between stages 1 and 2. ${ }^{a}$ Comparison the $U C / C D$ ratio between stages 1,2 , and 3 .

Table 4. Clinical Features of Patients with UC in 2001 to 2015

\begin{tabular}{|c|c|c|c|c|c|}
\hline UC & Over all & $\begin{array}{c}\text { Stage } 1 \\
(2001-2005)\end{array}$ & $\begin{array}{c}\text { Stage } 2 \\
(2006-2010)\end{array}$ & $\begin{array}{c}\text { Stage } 3 \\
(2011-2015)\end{array}$ & $P$-value \\
\hline No. of cases & 2,887 & 886 & 835 & 1,166 & - \\
\hline \multicolumn{6}{|l|}{ Age (yr) } \\
\hline Mean & $44.94 \pm 15.61$ & $45.41 \pm 15.92$ & $44.65 \pm 15.44$ & $44.82 \pm 15.58$ & 0.5654 \\
\hline Median (01-03) & $44(34-55)$ & $44(34-56)$ & 43 (34-55) & $45(33-56)$ & \\
\hline \multicolumn{6}{|l|}{ Peak age (top 5 age, \%) } \\
\hline & $38(2.99)$ & $34(3.05)$ & $38(3.47)$ & $49(3.00)$ & \\
\hline & $43(2.74)$ & $38(3.05)$ & $37(3.11)$ & $47(2.92)$ & \\
\hline & $49(2.71)$ & $40(2.93)$ & $49(3.11)$ & $43(2.74)$ & \\
\hline & $42(2.68)$ & $43(2.93)$ & $39(2.99)$ & $32(2.49)$ & \\
\hline & $47(2.62)$ & $42(2.82)$ & $42(2.99)$ & $41(2.49)$ & \\
\hline Age groups & & & & & 0.3793 \\
\hline$<20$ & 115 (3.98) & $33(3.72)$ & $31(3.71)$ & $51(4.37)$ & \\
\hline $20-39$ & $1,003(34.74)$ & 306 (34.54) & $314(37.60)$ & $383(32.85)$ & \\
\hline $40-59$ & $1,251(43.33)$ & $373(42.10)$ & $353(42.28)$ & $525(45.03)$ & \\
\hline $60-79$ & 477 (16.52) & $160(18.06)$ & $124(14.85)$ & $193(16.55)$ & \\
\hline$\geq 80$ & $41(1.42)$ & $14(1.58)$ & $13(1.56)$ & $14(1.20)$ & \\
\hline Male/female ratio & 1.62 & 1.63 & 1.41 & 1.80 & 0.0361 \\
\hline Prevalence $(1 / 100,000)$ & 7.62 & 3.70 & 7.68 & 11.36 & $<0.0001$ \\
\hline Incidence $(1 / 100,000)$ & 0.84 & 0.79 & 0.94 & 0.79 & $<0.0001$ \\
\hline
\end{tabular}

Values are presented as mean \pm SD, median (range), or number (\%). 


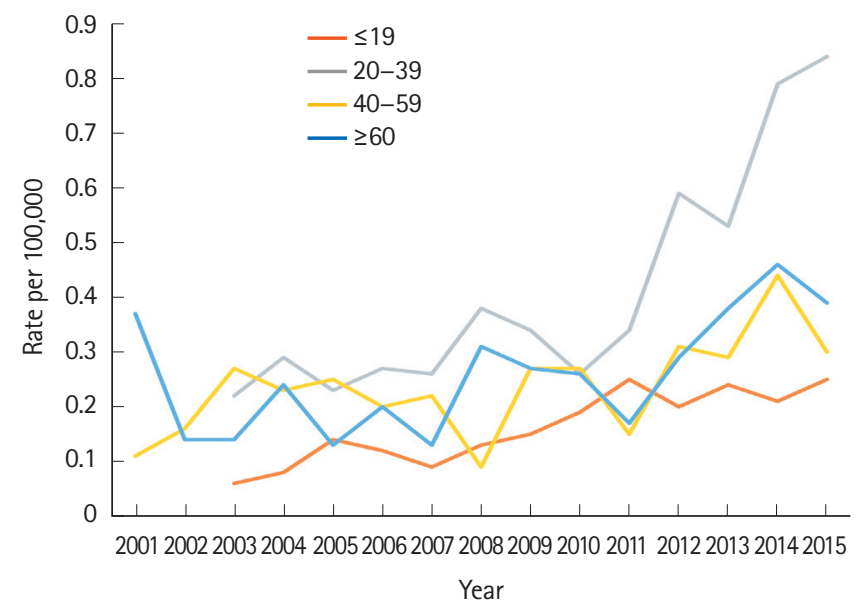

Fig. 4. Incidence of CD from 2001 to 2015 stratified by patient age.

of patients at registration for IBD, CD, and UC over the 3 stages. To identify which subpopulations exhibited the most significant increases in CD and UC registrations during the study period, we compared the incidence of CD and UC in 4 age groups ( $\leq 19,20-39,40-59$, and $\geq 60$ years) from 2001 to 2015 . We identified a significantly greater increase in the incidence rate among the 20 to 39 years group $(P<0.0001)$ compared with the other age groups (Fig. 6).

\section{DISCUSSION}

Analyzing data from the nationwide insurance registration system, we demonstrated that the incidence and prevalence of IBD increased steadily from 2001 to 2015 in Taiwan. Globally, the incidence level of IBD is highest in Western countries, with annual rates as high as 24.3 people per 100,000 for UC and 29.3 people per 100,000 for $\mathrm{CD}^{1,2,7}$ The present study revealed that the incidence rates of UC $(0.95 / 100,000)$ and CD $(0.47 / 100,000)$ have been increasing in Taiwan since the beginning of the 21st century. The incidence rates in Taiwan are low compared with those in Japan, South Korea, ${ }^{19}$ and Hong $\mathrm{Kong}^{7,12}$ but are similar to those in other Asian countries such as Singapore, Malaysia, and Thailand. ${ }^{1}$ Differences in degree of urbanization and socioeconomic status among the populations of these regions may have contributed to the described variation. ${ }^{5}$ In the present study, incidence levels may have been underestimated, because we used relatively stringent criteria for analysis. In studies of other countries, most incidence and prevalence levels have been calculated using clinical-diagnosis data only. By contrast, incidence and prevalence levels in this study were based on numbers of patients who

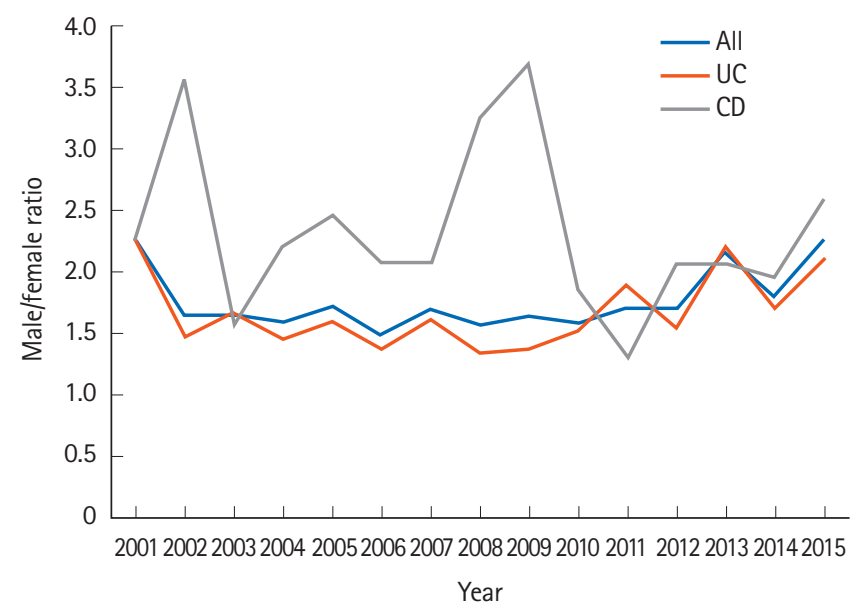

Fig. 5. Sex ratio (male to female) of patients with IBD in Taiwan from 2001 to 2015.

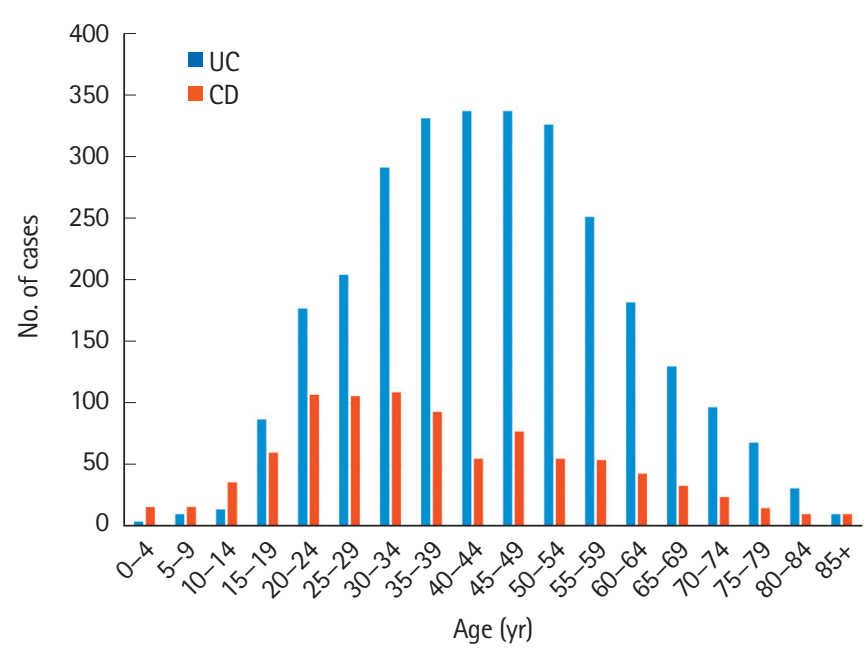

Fig. 6. Age distribution of patients with CD and UC from 2001 to 2015 in Taiwan.

had completed catastrophic-illness registration, which requires clinical records, endoscopic images, and pathology results.

In this study, we first demonstrated a decrease in the UC-toCD incidence ratio in Taiwan. Decreases have also been observed in other low-incidence Asian countries such as Hong Kong (from 8.94 in 1981-1985 to 1.03 in 2011-2014), ${ }^{12}$ South Korea (from 6.8 in 1986-1990 to 2.3 in 2001-2005), ${ }^{13}$ and Malaysia (from 8:1 in 1990-2000 to 3.6:1 in 2000-2010). ${ }^{14}$ By contrast, the UC-to-CD ratio has remained steady in Western countries (Fig. 7). ${ }^{9,12,13,19,22-24}$ Consistent with other studies from developing countries, we demonstrated that UC remains the predominant IBD in Asia and is twice as common as CD. Historically, the incidence of UC has usually increased first, followed by that of CD. ${ }^{2}$ For instance, a study conducted in Den- 


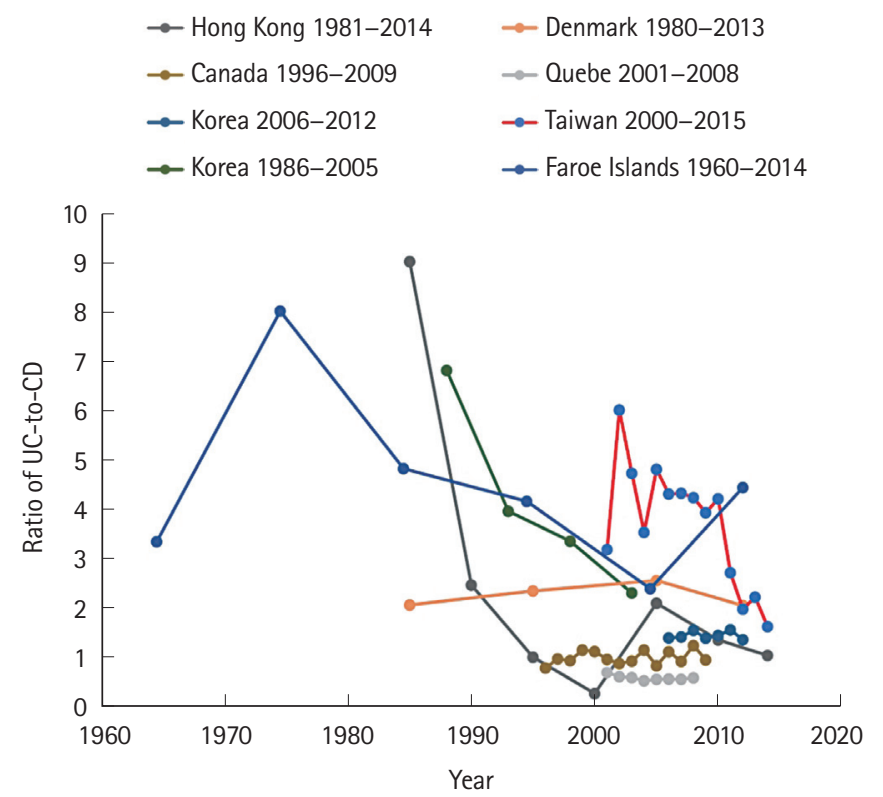

Fig. 7. Temporal trends in the UC-to-CD ratio from populationbased studies in Western and Asian countries. Data Sources: Hong Kong (1981-2014), ${ }_{1}^{12}$ Canada (1996-2009), ${ }_{1}^{22}$ Korea (20062012), ${ }_{1}^{19}$ Korea (1986-2005), ${ }_{1}^{13}$ Denmark (1980-2013) ${ }_{1}^{23}$ Quebec (2001-2008), ${ }^{24}$ the Faroe Islands (1960-2014), ${ }^{9}$ Taiwan (20012015): present study.

mark ${ }^{10}$ revealed a steady increase in CD incidence alongside a stable UC incidence from 1981 to 1992. In Japan, the incidence rate of UC peaked in 1974 whereas that of CD peaked in 1979. ${ }^{11}$ A similar trend was observed in the present study: in Taiwan, UC-to-CD ratio significantly decreased during the study period, from 4.32 (2001-2005) to 4.17 (2006-2010) to $2.03(2011-2015)$.

The reason for the catch-up of CD incidence between Taiwan and countries with higher incidence levels is not clear, but the trend may reflect common risk factors for CD and UC among various populations, such as improved social economic status and diet. The time lag between increases in UC and $\mathrm{CD}$ incidences may imply that the pathogeneses of the diseases differ ${ }^{12}$ or may reflect improvements in CD-diagnosis accuracy. ${ }^{9}$ The diagnosis of UC is usually straightforward and based on endoscopic findings from sigmoidoscopy. ${ }^{20} \mathrm{By}$ contrast, the diagnosis of $\mathrm{CD}$ is more complex and sometimes requires multiple evaluations, including enteroscopic, pathologic, and radiologic evaluations. ${ }^{21}$ We speculate that the improved endoscopic technology of the 21st century-that is, capsule endoscopy and deep enteroscopy ${ }^{25}$-may have enabled earlier diagnosis of CD. This phenomenon may partially explain the relatively steep increases in the $\mathrm{CD}$ incidence rate in most Asian countries in the 21 st century. ${ }^{26}$

In the present study, the most common age of registration of patients with CD was between 20 and 39 years, whereas that for UC was between 40 and 59 years. The mean age of patients registered with CD and UC was compared with that from reports conducted in other countries in Asia. ${ }^{20,21} \mathrm{Al}-$ though the results of the present study revealed that the median age of patient IBD registration did not significantly change during 2001 to 2015, a significant increase in the CD incidence rate was discovered among 20 to 39-year-old. Additionally, we found that the previously identified male predominance continued, with the male-to-female ratio not changing from 2001 to 2015 .

Our findings may have several implications for medical practice in Taiwan as well as in other Asian countries with low IBD incidence. First, similar to the increased incidence of IBD, an increased incidence of colorectal cancer has been observed in Taiwan, and the need for colonoscopy screening has increased. ${ }^{27}$ Thus, endoscopists must learn to diagnose colorectal neoplasm and IBD early to improve patient outcomes. Specifically, endoscopists with experience in IBD care must diagnose IBD early, differentiate among instances of IBD and infectious colitis, and help patients manage IBD-related complications. The Taiwan Society of Inflammatory Bowel Disease (TSIBD) was founded in 2013 with the aim of improving awareness, quality of care, and outcomes for patients with IBD in Taiwan. ${ }^{20,21}$ Second, the high cost of caring for patients with IBD combined with the increased incidence of IBD may have a considerable financial effect on the health care system. ${ }^{28}$ The use of biologic therapy has been suggested to reduce requirements for surgery and hospitalization and to improve quality of life and work productivity among patients with IBD. ${ }^{29,30} \mathrm{How}$ ever, the Taiwan health insurance criteria for biologic-therapy coverage and coverage period are more stringent than those for most other countries discussed herein, such as Japan and South Korea. ${ }^{30}$ Therefore, the TSIBD multicenter IBD registry study ${ }^{31}$ was initiated to obtain precise data on IBD patient clinical information, disease phenotype, and treatment strategies and outcomes. These data may eventually prompt the health care system to cover more expenses for patients with TBD.

Some of the factors associated with IBD incidence may also be associated with changes in disease severity over time. For example, IBD in Western countries may still be more severe than in Asia. Analyzing the incidence trend of IBD may provide clues regarding IBD pathogenesis. ${ }^{3}$ Policy planners and taxpayers should understand the global burden of IBD. Proof 
that IBD has a considerable disease burden may affect research funding allocated by national authorities.

This study has some intrinsic limitations from registration data. First, it is possible that a portion of IBD patients were not registered with catastrophic illness and not included in the current study. Second, our catastrophic illnesses registration permitted either UC or CD but not IBD-unclassified. Third, there was also no detail data regarding the disease location, activity, patterns of drugs used for patients included in this study. Fourth, there is no firm diagnostic criteria of UC and CD in Taiwan. The NHI reviewer usually give certification of IBD with reference to the Taiwan consensus for $\mathrm{UC}^{20}$ and $\mathrm{CD}^{21}$

In conclusion, using Taiwan's nationwide insurance database to identify patients with IBD, the present study revealed that the incidence and prevalence of IBD in Taiwan steadily increased from 2001 to 2015. The number of patients with CD increased more rapidly than that of patients with UC. Although the incidence of IBD remains low in Taiwan, the upward trend in CD incidence in Taiwan as well as other developed countries suggests that requirements for IBD care will be greater in the future.

\section{FINANCIAL SUPPORT}

This work was supported by the National Taiwan University Hospital Research Program Ms160 (to JMW), 107-A139 (to SCW). and the Liver Disease Prevention and Treatment Research Foundation, Taiwan (to MTW and SCW).

\section{CONFLICT OF INTEREST}

No potential conflict of interest relevant to this article was reported.

\section{AUTHOR CONTRIBUTION}

Yen HH: data analysis and manuscript drafting; Weng MT, Tung CC, Shieh MJ, Wong JM: data analysis and drafting of the Results and Discussion sections; Wang YT, Chang YT, Chang CH: data and statistical analyses; Wei SC: study design, data collection, data analysis, and manuscript drafting.

\section{ACKNOWLEDGEMENTS}

We would like to express gratitude to the individuals associated with the Second Core Laboratory and the Integrated data- base of the Department of Medical Research of the National Taiwan University Hospital for technical assistance.

\section{REFERENCES}

1. Ng SC, Bernstein CN, Vatn MH, et al. Geographical variability and environmental risk factors in inflammatory bowel disease. Gut 2013;62:630-649.

2. Chi KR. Epidemiology: rising in the east. Nature 2016;540: S100-S102.

3. Ng SC, Shi HY, Hamidi N, et al. Worldwide incidence and prevalence of inflammatory bowel disease in the 21st century: a systematic review of population-based studies. Lancet 2018;390:2769-2778.

4. Kaplan GG. The global burden of IBD: from 2015 to 2025 . Nat Rev Gastroenterol Hepatol 2015;12:720-727.

5. Wei SC, Lin MH, Tung CC, et al. A nationwide populationbased study of the inflammatory bowel diseases between 1998 and 2008 in Taiwan. BMC Gastroenterol 2013;13:166.

6. Ishige T, Tomomasa T, Hatori R, et al. Temporal trend of pediatric inflammatory bowel disease: analysis of national registry data 2004-2013 in Japan. J Pediatr Gastroenterol Nutr 2017; 65:e80-e82.

7. Ng WK, Wong SH, Ng SC. Changing epidemiological trends of inflammatory bowel disease in Asia. Intest Res 2016;14:111119.

8. Yang H, Li Y, Wu W, et al. The incidence of inflammatory bowel disease in Northern China: a prospective population-based study. PLoS One 2014;9:e101296.

9. Hammer T, Nielsen KR, Munkholm P, Burisch J, Lynge E. The Faroese IBD study: incidence of inflammatory bowel diseases across 54 years of population-based data. J Crohns Colitis 2016;10:934-942.

10. Fonager K, Sørensen HT, Olsen J. Change in incidence of Crohn's disease and ulcerative colitis in Denmark: a study based on the national registry of patients, 1981-1992. Int J Epidemiol 1997;26:1003-1008.

11. Yoshida Y, Murata Y. Inflammatory bowel disease in Japan: studies of epidemiology and etiopathogenesis. Med Clin North Am 1990;74:67-90.

12. Ng SC, Leung WK, Shi HY, et al. Epidemiology of inflammatory bowel disease from 1981 to 2014: results from a territorywide population-based registry in Hong Kong. Inflamm Bowel Dis 2016;22:1954-1960.

13. Yang SK, Yun S, Kim JH, et al. Epidemiology of inflammatory bowel disease in the Songpa-Kangdong district, Seoul, Korea, 
1986-2005: a KASID study. Inflamm Bowel Dis 2008;14:542549.

14. Hilmi I, Jaya F, Chua A, Heng WC, Singh H, Goh KL. A first study on the incidence and prevalence of IBD in Malaysia: results from the Kinta Valley IBD Epidemiology Study. J Crohns Colitis 2015;9:404-409.

15. Prideaux L, Kamm MA, De Cruz PP, Chan FK, Ng SC. Inflammatory bowel disease in Asia: a systematic review. J Gastroenterol Hepatol 2012;27:1266-1280.

16. Ng SC, Tsoi KK, Kamm MA, et al. Genetics of inflammatory bowel disease in Asia: systematic review and meta-analysis. Inflamm Bowel Dis 2012;18:1164-1176.

17. Bernstein CN, Shanahan F. Disorders of a modern lifestyle: reconciling the epidemiology of inflammatory bowel diseases. Gut 2008;57:1185-1191.

18. Wei SC, Shieh MJ, Chang MC, Chang YT, Wang CY, Wong JM. Long-term follow-up of ulcerative colitis in Taiwan. J Chin Med Assoc 2012;75:151-155.

19. Kim HJ, Hann HJ, Hong SN, et al. Incidence and natural course of inflammatory bowel disease in Korea, 2006-2012: a nationwide population-based study. Inflamm Bowel Dis 2015;21:623-630.

20. Wei SC, Chang TA, Chao TH, et al. Management of ulcerative colitis in Taiwan: consensus guideline of the Taiwan Society of Inflammatory Bowel Disease. Intest Res 2017;15:266-284.

21. Wei SC, Chang TA, Chao TH, et al. Management of Crohn's disease in Taiwan: consensus guideline of the Taiwan Society of Inflammatory Bowel Disease. Intest Res 2017;15:285-310.

22. Leddin D, Tamim H, Levy AR. Decreasing incidence of inflammatory bowel disease in eastern Canada: a population database study. BMC Gastroenterol 2014;14:140.
23. Lophaven SN, Lynge E, Burisch J. The incidence of inflammatory bowel disease in Denmark 1980-2013: a nationwide cohort study. Aliment Pharmacol Ther 2017;45:961-972.

24. Bitton A, Vutcovici M, Patenaude V, Sewitch M, Suissa S, Brassard P. Epidemiology of inflammatory bowel disease in Québec: recent trends. Inflamm Bowel Dis 2014;20:1770-1776.

25. Yen HH, Chang CW, Chou JW, Wei SC. Balloon-assisted enteroscopy and capsule endoscopy in suspected small bowel Crohn's disease. Clin Endosc 2017;50:417-423.

26. Kaplan GG, Ng SC. Understanding and preventing the global increase of inflammatory bowel disease. Gastroenterology 2017;152:313-321.

27. Chiang TH, Lee YC, Liao WC, et al. Timing and risk factors for a positive fecal immunochemical test in subsequent screening for colorectal neoplasms. PLoS One 2015;10:e0136890.

28. van der Valk ME, Mangen MJ, Leenders M, et al. Healthcare costs of inflammatory bowel disease have shifted from hospitalisation and surgery towards anti-TNFalpha therapy: results from the COIN study. Gut 2014;63:72-79.

29. Wei SC. Differences in the public medical insurance systems for inflammatory bowel disease treatment in Asian countries. Intest Res 2016;14:218-223.

30. Chang CW, Wei SC, Chou JW, et al. Safety and efficacy of adalimumab for patients with moderate to severe Crohn's disease: the Taiwan Society of Inflammatory Bowel Disease (TSIBD) Study. Intest Res 2014;12:287-292.

31. Lin WC, Chou JW, Yen HH, et al. Outcomes of limited period of adalimumab treatment in moderate to severe Crohn's disease patients: Taiwan Society of Inflammatory Bowel Disease Study. Intest Res 2017;15:487-494. 